\title{
ON EISENSTEIN-DUMAS AND GENERALIZED SCHÖNEMANN POLYNOMIALS
}

\author{
Anuj Bishnoi and Sudesh K. Khanduja* \\ Department of Mathematics, Panjab University, Chandigarh-160014, India. \\ E-mail: anuj.bshn@gmail.com, skhand@pu.ac.in
}

\begin{abstract}
Let $v$ be a valuation of a field $K$ having value group $\mathbb{Z}$. It is known that a polynomial $x^{n}+a_{n-1} x^{n-1}+\ldots+a_{0}$ satisfying $\frac{v\left(a_{i}\right)}{n-i} \geqslant \frac{v\left(a_{0}\right)}{n}>0$ with $v\left(a_{0}\right)$ coprime to $n$, is irreducible over $K$. Such a polynomial is referred to as an Eisenstein-Dumas polynomial with respect to $v$. In this paper, we give necessary and sufficient conditions so that some translate $g(x+a)$ of a given polynomial $g(x)$ belonging to $K[x]$ is an Eisenstein-Dumas polynomial with respect to $v$. In fact an analogous problem is dealt with for a wider class of polynomials, viz. Generalized Schönemann polynomials with coefficients over valued fields of arbitrary rank.
\end{abstract}

Keywords : Field Theory and polynomials; Valued fields; Non-Archimedean valued fields.

2000 Mathematics Subject Classification : 12E05; 12J10; 12J25.

*All correspondence may be addressed to this author. 


\section{INTRODUCTION}

The classical Schönemann Irreducibility Criterion states that if $f(x)$ is a monic polynomial with coefficients from the ring $\mathbb{Z}$ of integers which is irreducible modulo a prime number $p$ and if $g(x)$ belonging to $\mathbb{Z}[x]$ is a polynomial of the form $g(x)=f(x)^{e}+p M(x)$ where $M(x)$ belonging to $\mathbb{Z}[x]$ is relatively prime to $f(x)$ modulo $p$ and the degree of $M(x)$ is less than the degree of $g(x)$, then $g(x)$ is irreducible over the field $\mathbb{Q}$ of rational numbers. Such a polynomial is referred to as a Schönemann polynomial with respect to $p$ and $f(x)$. It can be easily seen that if $g(x)$ is as above, then the $f(x)$-expansion of $g(x)$ obtained on dividing it by successive powers of $f(x)$ given by

$$
g(x)=\sum_{i=0}^{e} g_{i}(x) f(x)^{i}, \operatorname{deg} g_{i}(x)<\operatorname{deg} f(x),
$$

satisfies (i) $g_{e}(x)=1$, (ii) $p$ divides the content of each polynomial $g_{i}(x)$ for $0 \leqslant i \leqslant e-1$ and (iii) $p^{2}$ does not divide the content of $g_{0}(x)$. Clearly any polynomial $g(x)$ belonging to $\mathbb{Z}[x]$ whose $f(x)$-expansion satisfies the above three properties is a Schönemann polynomial with respect to $p$ and $f(x)$. Note that a monic polynomial is an Eisenstein polynomial with respect to a prime $p$ if and only if it is a Schönemann polynomial with respect to $p$ and $f(x)=x$.

The Schönemann Irreducibility Criterion has been extended to polynomials with coefficients over valued fields in several ways (cf. Khanduja and Saha, 1997; Ribenboim, 1999, Chapter 4, D; Brown, 2008). In 2008, Ron Brown gave a generalization of the Schönemann Irreducibility Criterion for polynomials with coefficients in a valued field $(K, v)$ of arbitrary rank, which will be stated after introducing some notations.

We shall denote by $v^{x}$ the Gaussian valuation of the field $K(x)$ of rational functions in an indeterminate $x$ which extends the valuation $v$ of $K$ and is defined on $K[x]$ by

$$
v^{x}\left(\sum_{i} a_{i} x^{i}\right)=\min _{i}\left\{v\left(a_{i}\right) \mid a_{i} \in K\right\} .
$$

For an element $\xi$ in the valuation ring $R_{v}$ of $v$ with maximal ideal $\mathcal{M}_{v}, \bar{\xi}$ will denote its $v$-residue, i.e., the image of $\xi$ under the canonical homomorphism from $R_{v}$ onto $R_{v} / \mathcal{M}_{v}$. For $f(x)$ belonging to $R_{v}[x], \bar{f}(x)$ will stand for the polynomial over $R_{v} / \mathcal{M}_{v}$ obtained by replacing each coefficient of $f(x)$ by its $v$-residue. The following result of Ron Brown which generalizes the Schönemann Irreducibility Criterion is proved in section 3 (see Lemma 3.1). 
Theorem A. Let $v$ be a valuation of arbitrary rank of a field $K$ with value group $G$ and valuation ring $R_{v}$ having maximal ideal $\mathcal{M}_{v}$. Let $f(x)$ belonging to $R_{v}[x]$ be a monic polynomial of degree $m$ such that $\bar{f}(x)$ is irreducible over $R_{v} / \mathcal{M}_{v}$. Assume that $g(x) \in R_{v}[x]$ is a monic polynomial whose $f(x)$-expansion $f(x)^{e}+\sum_{i=0}^{e-1} g_{i}(x) f(x)^{i}$ satisfies $\frac{v^{x}\left(g_{i}(x)\right)}{e-i} \geqslant \frac{v^{x}\left(g_{0}(x)\right)}{e}>0$ for $0 \leqslant i \leqslant e-1$ and $v^{x}\left(g_{0}(x)\right) \notin d G$ for any number $d>1$ dividing e. Then $g(x)$ is irreducible over $K$.

A polynomial satisfying the hypothesis of Theorem A will be referred to as a Generalized Schönemann polynomial with respect to $v$ and $f(x)$. In the particular case when $f(x)=x$, it will be called an Eisenstein-Dumas polynomial with respect to $v$. When $v$ is a discrete valuation with value group $\mathbb{Z}$, then a monic polynomial $\sum_{i=0}^{e} a_{i} x^{i}$ is an Eisenstein-Dumas polynomial with respect to $v$ if $\frac{v\left(a_{i}\right)}{e-i} \geqslant \frac{v\left(a_{0}\right)}{e}$ and $v\left(a_{0}\right)$ is coprime to $e$. Thus Theorem A extends the usual Eisenstein-Dumas Irreducibility Criterion ${ }^{1}$.

In this paper, we first investigate when a translate $g(x+a)$ of a given polynomial $g(x)$ belonging to $K[x]$ having a root $\theta$ is an Eisenstein-Dumas polynomial with respect to an arbitrary henselian valuation $v$ of a field $K$. It is shown that $g(x+a)$ is such a polynomial if and only if $K(\theta) / K$ is a totally ramified extension and $(\theta, a)$ is a $(K, v)$-distinguished pair as defined below. In particular, it is deduced that if some translate of a polynomial $g(x)=x^{e}+a_{e-1} x^{e-1}+\ldots+a_{0}$ is an Eisenstein-Dumas polynomial with respect to $v$ with $e$ not divisible by the characteristic of the residue field of $v$, then the polynomial $g\left(x-\frac{a_{e-1}}{e}\right)$ is an Eisenstein-Dumas polynomial with respect to $v$. This generalizes a result of M. Juras proved in 2006 (cf. Juras, 2006).

We also deal with the following more general problem related to Theorem A.

Let $g(x)$ belonging to $R_{v}[x]$ be a monic polynomial over a henselian valued field $(K, v)$ of arbitrary rank with $\bar{g}(x)=\phi(x)^{e}$ where $\phi(x)$ is an irreducible polynomial over $R_{v} / \mathcal{M}_{v}$ and $\theta$ is a root of $g(x)$. What are necessary and sufficient conditions so that $g(x)$ is a Generalized Schönemann polynomial with respect to $v$ and some polynomial $f(x) \in R_{v}[x]$ with $\bar{f}(x)=\phi(x)$ ?

\footnotetext{
${ }^{1}$ Eisenstein-Dumas Irreducibility Criterion. Let $g(x)=a_{n} x^{n}+a_{n-1} x^{n-1}+\ldots+a_{o}$ be a polynomial with coefficients in $\mathbb{Z}$. Suppose there exists a prime $p$ whose exact power $p^{r_{i}}$ dividing $a_{i}$ (where $r_{i}=\infty$ if $\left.a_{i}=0\right)$, satisfy $r_{n}=0,\left(r_{i} / n-i\right) \geqslant\left(r_{0} / n\right)$ for $0 \leqslant i \leqslant n-1$ and $r_{0}, n$ are coprime. Then $g(x)$ is irreducible over $\mathbb{Q}$.
} 
Our results are proved using saturated distinguished chains which will be defined after introducing some notations.

In what follows, $v$ is a henselian valuation of arbitrary rank of a field $K$ and $\tilde{v}$ is the unique prolongation of $v$ to the algebraic closure $\widetilde{K}$ of $K$ with value group $\widetilde{G}$. By the degree of an element $\alpha$ in $\widetilde{K}$, we shall mean the degree of the extension $K(\alpha) / K$ and shall denote it by $\operatorname{deg} \alpha$. For an element $\xi$ in the valuation ring of $\tilde{v}, \bar{\xi}$ will stand for its $\tilde{v}$-residue and for a subfield $L$ of $\widetilde{K}, \bar{L}$ and $G(L)$ will denote respectively the residue field and the value group of the valuation of $L$ obtained by restricting $\tilde{v}$. When there is no chance of confusion, we shall write $\tilde{v}(\alpha)$ as $v(\alpha)$ for $\alpha$ belonging to $\widetilde{K}$.

A finite extension $\left(K^{\prime}, v^{\prime}\right) /(K, v)$ is called defectless if $\left[K^{\prime}: K\right]=e f$, where $e$ and $f$ are the index of ramification and the residual degree of $v^{\prime} / v$.

Recall that a pair $(\theta, \alpha)$ of elements of $\widetilde{K}$ is called a distinguished pair (more precisely $(K, v)$-distinguished pair) if the following three conditions are satisfied: (i) $\operatorname{deg} \theta>\operatorname{deg} \alpha$, (ii) $\tilde{v}(\theta-\beta) \leqslant \tilde{v}(\theta-\alpha)$ for every $\beta$ in $\widetilde{K}$ with $\operatorname{deg} \beta<\operatorname{deg} \theta$, (iii) if $\gamma \in \widetilde{K}$ and $\operatorname{deg} \gamma<\operatorname{deg} \alpha$, then $\tilde{v}(\theta-\gamma)<\tilde{v}(\theta-\alpha)$.

Distinguished pairs give rise to distinguished chains in a natural manner. A chain $\theta=\theta_{0}, \theta_{1}, \ldots, \theta_{r}$ of elements of $\widetilde{K}$ will be called a saturated distinguished chain for $\theta$ if $\left(\theta_{i}, \theta_{i+1}\right)$ is a distinguished pair for $0 \leqslant i \leqslant r-1$ and $\theta_{r} \in K$. Popescu and Zaharescu (cf. Popescu and Zaharescu, 1995) were the first to introduce the notion of distinguished chains. In 1995, they proved the existence of a saturated distinguished chain for each $\theta$ belonging to $\widetilde{K} \backslash K$ in case $(K, v)$ is a complete discrete rank one valued field. In 2005, Aghigh and Khanduja (cf. Aghigh and Khanduja, 2005) proved that if $(K, v)$ is a henselian valued field of arbitrary rank, then an element $\theta$ belonging to $\widetilde{K} \backslash K$ has a saturated distinguished chain with respect to $v$ if and only if $K(\theta)$ is a defectless extension of $(K, v)$. A saturated distinguished chain for $\theta$ gives rise to several invariants associated with $\theta$, some of which are given by Theorem B stated below which is proved in (cf. Aghigh and Khanduja, 2005).

Theorem B. Let $(K, v)$ and $(\widetilde{K}, \tilde{v})$ be as above. Let $\theta=\theta_{0}, \theta_{1}, \ldots, \theta_{r}$ and $\theta=\eta_{0}, \eta_{1}, \ldots, \eta_{s}$ be two saturated distinguished chains for an element $\theta$ belonging to $\widetilde{K} \backslash K$, then $r=s$ and $\left[K\left(\theta_{i}\right): K\right]=\left[K\left(\eta_{i}\right): K\right], G\left(K\left(\theta_{i}\right)\right)=G\left(K\left(\eta_{i}\right)\right)$, $\overline{K\left(\theta_{i}\right)}=\overline{K\left(\eta_{i}\right)}$ for $1 \leqslant i \leqslant r$. Further $G\left(K\left(\theta_{i+1}\right)\right) \subseteq G\left(K\left(\theta_{i}\right)\right), \overline{K\left(\theta_{i+1}\right)} \subseteq \overline{K\left(\theta_{i}\right)}$ for $0 \leqslant i \leqslant r-1$. 
In this paper, we prove

Theorem 1.1. Let $v$ be a henselian valuation of arbitrary rank of a field $K$ with value group $G$. Let $g(x)$ belonging to $R_{v}[x]$ be a monic polynomial of degree $e$ having a root $\theta$. Then for an element a of $K, g(x+a)$ is an EisensteinDumas polynomial with respect to $v$ if and only if $(\theta, a)$ is a distinguished pair and $K(\theta) / K$ is a totally ramified extension of degree $e$.

The following result which generalizes a result of M. Juras will be quickly deduced from the above theorem.

Theorem 1.2. Let $g(x)=\sum_{i=0}^{e} a_{i} x^{i}$ be a monic polynomial with coefficients in a henselian valued field $(K, v)$. Suppose that the characteristic of the residue field of $v$ does not divide $e$. If there exists an element $b$ belonging to $K$ such that $g(x+b)$ is an Eisenstein-Dumas polynomial with respect to $v$, then so is $g\left(x-\frac{a_{e-1}}{e}\right)$.

Note that if $g(x)$ belonging to $R_{v}[x]$ is a monic polynomial such that $\bar{g}(x)$ is irreducible over $R_{v} / \mathcal{M}_{v}$, then for any non-zero $\alpha$ in $\mathcal{M}_{v}, g(x)$ is a Generalized Schönemann polynomial with respect to $f(x)=g(x)-\alpha$ and $v$. Therefore to deal with the second problem mentioned after Theorem A, we may assume that $\bar{g}(x)=\phi(x)^{e}$ with $\phi(x)$ irreducible over $R_{v} / \mathcal{M}_{v}$ and $e>1$. When $\operatorname{deg} \phi(x)=1$ then the problem referred to above is already solved in Theorem 1.1 because $g(x+a)$ is an Eisenstein-Dumas polynomial with respect to $v$ if and only if $g(x)$ is a Generalized Schönemann polynomial with respect to $v$ and $x-a$. Setting aside these two cases, we shall prove

Theorem 1.3. Let $v$ be a henselian valuation of arbitrary rank of a field $K$ with value group $G$ and $f(x)$ belonging to $R_{v}[x]$ be a monic polynomial of degree $m>1$ with $\bar{f}(x)$ irreducible over the residue field of $v$. Let $g(x) \in K[x]$ be a Generalized Schönemann polynomial with respect to $v$ and $f(x)$ having $f(x)$ expansion $f(x)^{e}+\sum_{i=0}^{e-1} g_{i}(x) f(x)^{i}$ with $e>1$. Let $\theta$ be a root of $g(x)$. Then for some suitable root $\theta_{1}$ of $f(x), \theta$ has a saturated distinguished chain $\theta=\theta_{0}, \theta_{1}, \theta_{2}$ of length 2 with $G\left(K\left(\theta_{1}\right)\right)=G, \overline{K(\theta)}=\bar{K}(\bar{\theta})$ and $[G(K(\theta)): G]=e$.

The converse of the above result is also true as asserted by the following theorem. 
Theorem 1.4. Let $(K, v)$ be as in the above theorem. Let $g(x)$ belonging to $R_{v}[x]$ be a monic polynomial such that $\bar{g}(x)=\phi(x)^{e}, e>1$ where $\phi(x)$ is an irreducible polynomial over $R_{v} / \mathcal{M}_{v}$ of degree $m>1$. Suppose that a root $\theta$ of $g(x)$ has a saturated distinguished chain $\theta=\theta_{0}, \theta_{1}, \theta_{2}$ of length 2 with $G\left(K\left(\theta_{1}\right)\right)=G$, $\overline{K(\theta)}=\bar{K}(\bar{\theta})$ and $[G(K(\theta)): G]=e$. Then $g(x)$ is a Generalized Schönemann polynomial with respect to $v$ and $f(x)$, where $f(x)$ is the minimal polynomial of $\theta_{1}$ over $K$.

\section{PROOF OF THEOREMS $1.1,1.2$}

Proof of Theorem 1.1. Write $g(x+a)=x^{e}+a_{e-1} x^{e-1}+\ldots+a_{0}, a, a_{i} \in K$. Suppose first that $g(x+a)$ is an Eisenstein-Dumas polynomial with respect to $v$. Then it is irreducible over $K$ in view of Theorem A. Since $(K, v)$ is henselian, all roots of $g(x+a)$ have the same $v$-valuation and hence $v(\theta-a)=\frac{v\left(a_{0}\right)}{e}$. In view of the hypothesis that $g(x+a)$ is an Eisenstein-Dumas polynomial, we have

$$
e=\left[G+\mathbb{Z} \frac{v\left(a_{0}\right)}{e}: G\right]=[G+\mathbb{Z} v(\theta-a): G]
$$

To prove that $(\theta, a)$ is a distinguished pair, it is to be shown that

$$
\max \{v(\theta-\beta) \mid \beta \in \widetilde{K}, \operatorname{deg} \beta<\operatorname{deg} \theta\}=v(\theta-a)=\frac{v\left(a_{0}\right)}{e} .
$$

If $\beta$ is as in (2) and if $v(\theta-\beta)>v(\theta-a)$, then by the strong triangle law,

$$
v(\beta-a)=\min \{v(\beta-\theta), v(\theta-a)\}=v(\theta-a)
$$

which in view of the fundamental inequality (cf. Engler and Prestel, 2005, Theorem 3.3.4) and (1) implies that $\operatorname{deg}(\beta-a) \geqslant e$, a contradiction. Therefore (2) holds and $(\theta, a)$ is a distinguished pair with $K(\theta) / K$ totally ramified in view of (1).

Conversely suppose that $(\theta, a)$ is a distinguished pair and $K(\theta) / K$ is a totally ramified extension of degree $e$. Note that $v(\theta-a) \geqslant v(\theta) \geqslant 0$. Keeping in mind that $(K, v)$ is henselian and the relation between the roots and coefficients of the the $K$-irreducible polynomial $g(x+a)=x^{e}+a_{e-1} x^{e-1}+\ldots+a_{0}$, we see that $v\left(a_{i}\right) \geqslant(e-i) v(\theta-a)=\left(\frac{e-i}{e}\right) v\left(a_{0}\right) \geqslant 0$. So $g(x+a)$ is an Eisenstein-Dumas polynomial with respect to $v$ once we show that $s \frac{v\left(a_{0}\right)}{e} \notin G$ for any positive number $s<e$.

Suppose to the contrary there exists a positive number $s<e$ such that 
$s \frac{v\left(a_{0}\right)}{e}=s v(\theta-a) \in G$, say $s v(\theta-a)=v(b), b \in K$. Since $K(\theta) / K$ is totally ramified, there exists $c$ in $K$ with $v(c)=0$ such that $\left.\overline{\left((\theta-a)^{s} / b\right.}\right)=\bar{c}$, which implies that

$$
v\left((\theta-a)^{s}-b c\right)>v(b) .
$$

Set $v(\theta-a)=\delta$ and $h(x)=(x-a)^{s}-b c$. Let $w$ denote the valuation of $\widetilde{K}(x)$ defined on $\widetilde{K}[x]$ by

$$
w\left(\sum_{i} c_{i}(x-a)^{i}\right)=\min _{i}\left\{\tilde{v}\left(c_{i}\right)+i \delta\right\}, c_{i} \in \widetilde{K} .
$$

Note that $w(h(x))=\min \{s \delta, v(b c)\}=v(b)$. This equality will contradict (3) thereby completing the proof of the theorem once we show that

$$
v(h(\theta))=w(h(x))
$$

To verify (4), write $h(x)=\prod_{i=1}^{s}\left(x-\beta^{(i)}\right)$. Keeping in mind that $h(x)$ belonging to $K[x]$ is a polynomial of degree $s<e$ and the fact that $(\theta, a)$ is a distinguished pair, we have $v\left(\theta-\beta^{(i)}\right) \leqslant v(\theta-a)$ for $1 \leqslant i \leqslant s$ and hence it can be easily seen that

$$
v\left(\theta-\beta^{(i)}\right)=\min \left\{v(\theta-a), v\left(a-\beta^{(i)}\right)\right\}=w\left(x-\beta^{(i)}\right) .
$$

On summing over $i$, the above equation gives (4).

Proof of Theorem 1.2. In view of Theorem 1.1, it is enough to prove that if $(\theta, b)$ is a distinguished pair, then so is $\left(\theta, \frac{-a_{e-1}}{e}\right)$. In fact it suffices to show that

$$
v\left(\theta+\frac{a_{e-1}}{e}\right) \geqslant v(\theta-b) .
$$

Let $\theta=\theta^{(1)}, \theta^{(2)}, \ldots, \theta^{(e)}$ denote the $K$-conjugates of $\theta$. Using the hypothesis $v(e)=0$, we have

$$
v\left(\theta+\frac{a_{e-1}}{e}\right)=v\left(e \theta+a_{e-1}\right)=v\left(e \theta-\sum_{i=1}^{e} \theta^{(i)}\right)=v\left(\sum_{i=2}^{e}\left(\theta-\theta^{(i)}\right)\right) ;
$$

consequently

$$
v\left(\theta+\frac{a_{e-1}}{e}\right) \geqslant \min _{i \geqslant 2}\left\{v\left(\theta-\theta^{(i)}\right)\right\}=v\left(\theta-\theta^{(2)}\right)(\text { say }) .
$$

Since $b \in K, v(\theta-b)=v\left(\theta^{(2)}-b\right)$ and hence $v\left(\theta-\theta^{(2)}\right) \geqslant v(\theta-b)$ which together with (6) proves (5) and hence the theorem. 
We use Theorem 1.1 to construct examples of totally ramified extensions $K(\theta) / K$ such that no translate of the minimal polynomial of $\theta$ over $K$ is an Eisenstein-Dumas polynomial with respect to $v$.

Notation. For $\alpha$ separable over $K$ of degree $>1, \omega_{K}(\alpha)$ will stand for the Krasner's constant defined by

$$
\omega_{K}(\alpha)=\max \left\{\tilde{v}\left(\alpha-\alpha^{\prime}\right) \mid \alpha^{\prime} \neq \alpha \text { runs over } K \text {-conjugates of } \alpha\right\} .
$$

Example 2.1. Let $K$ be the field of 2-adic numbers with the usual valuation $v_{2}$ given by $v_{2}(2)=1$. The prolongation of $v_{2}$ to the algebraic closure of $K$ will be denoted by $v_{2}$ again. Consider $\theta=2+2\left(2^{-1 / 2}\right)+2^{2}\left(2^{-1 / 2^{2}}\right)$ and $\theta_{1}=2+2\left(2^{-1 / 2}\right)$. It will be shown that $K(\theta)=K\left(2^{1 / 4}\right)$ and $\left(\theta, \theta_{1}\right)$ is a distinguished pair. Note that the Krasner's constant $\omega_{K}\left(\theta_{1}\right)=3 / 2$ and $v_{2}\left(\theta-\theta_{1}\right)=7 / 4>\omega_{K}\left(\theta_{1}\right)$. Therefore by Krasner's Lemma (cf. Engler and Prestel, 2005, Theorem 4.1.7), $K\left(\theta_{1}\right) \subseteq K(\theta)$ and hence $2^{2}\left(2^{-1 / 4}\right)=\theta-\theta_{1}$ belongs to $K(\theta)$ as asserted. To show that $\left(\theta, \theta_{1}\right)$ is a distinguished pair, we first verify that whenever $\gamma$ belonging to $\widetilde{K}$ satisfies $v_{2}(\theta-\gamma)>v_{2}\left(\theta-\theta_{1}\right)=7 / 4$, then $\operatorname{deg} \gamma \geqslant 4$. If $\gamma$ is as above, we have by the strong triangle law

$$
v_{2}\left(\theta_{1}-\gamma\right)=\min \left\{v_{2}\left(\theta_{1}-\theta\right), v_{2}(\theta-\gamma)\right\}=7 / 4>\omega_{K}\left(\theta_{1}\right)=3 / 2 .
$$

So by Krasner's Lemma, $K\left(\theta_{1}\right) \subseteq K(\gamma)$ and hence $G(K(\gamma))$ contains $v_{2}\left(\theta_{1}-\gamma\right)=$ $7 / 4$ which implies that $\operatorname{deg} \gamma \geqslant 4$. Therefore

$$
7 / 4=v_{2}\left(\theta-\theta_{1}\right)=\max \left\{v_{2}(\theta-\beta) \mid \beta \in \widetilde{K}, \operatorname{deg} \beta<4\right\}
$$

Also for any $b \in \widetilde{K}$ with $\operatorname{deg} b<\operatorname{deg} \theta_{1}$, we have $b \in K$ and clearly $v_{2}\left(\theta_{1}-b\right) \leqslant$ $1 / 2<v_{2}\left(\theta-\theta_{1}\right)$. So $\left(\theta, \theta_{1}\right)$ is a distinguished pair. As can be easily checked, $\theta$ is a root of $g(x)=x^{4}-8 x^{3}+20 x^{2}-80 x+4$ which must be irreducible over $K$. By Theorem 1.1 no translate of $g(x)$ can be an Eisenstein-Dumas polynomial with respect to $v_{2}$ because $\left(\theta, \theta_{1}\right)$ is a distinguished pair with $\theta_{1} \notin K$ and consequently $(\theta, a)$ cannot be a distinguished pair for any $a$ in $K$ in view of Theorem B. Moreover, if $p$ be a prime number different from 2, then no translate of $g(x)$ can be an Eisenstein-Dumas polynomial with respect to the $p$-adic valuation $v_{p}$, for otherwise in view of Theorem 1.2, $g(x+2)=x^{4}-4 x^{2}-64 x-124$ would be an Eisenstein-Dumas polynomial with respect to $v_{p}$, which is clearly impossible.

\section{PROOF OF THEOREM 1.3.}

We need the following lemma which is proved in (cf. Brown, 2008, Lemma 4). Its proof is omitted. 
Lemma 3.1. Let $v, G, f(x)$ and $g(x)$ be as in Theorem $A$. Let $\theta$ be a root of $g(x)$ and $v^{\prime}$ be a prolongation of $v$ to $K(\theta)$ with value group $G^{\prime}$. Then $v^{\prime}(f(\theta))=$ $\frac{v^{x}\left(g_{0}(x)\right)}{e}, G^{\prime}=G+\mathbb{Z} \frac{v^{x}\left(g_{0}(x)\right)}{e}$, the residue field of $v^{\prime}$ is a simple extension of the residue field of $v$ generated by the $v^{\prime}$-residue $\bar{\theta}$ of $\theta$ and $g(x)$ is irreducible over $K$. In particular the index of ramification and the residual degree of $v^{\prime} / v$ are $e$ and deg $f(x)$ respectively.

Proof of Theorem 1.3. Since $\bar{\theta}$ is a root of $\bar{g}(x)=\bar{f}(x)^{e}$ and $\bar{f}(x)$ is an irreducible polynomial of degree $m>1$, it follows that $v(\theta)=0$ and there exists a root $\alpha^{(i)}$ of $f(x)$ such that $v\left(\theta-\alpha^{(i)}\right)>0$. Let $\alpha$ be a root of $f(x)$ satisfying

$$
0<v(\theta-\alpha)=\max \left\{v\left(\theta-\alpha^{(i)}\right) \mid \alpha^{(i)} \text { runs over roots of } f(x)\right\}=\delta(\text { say }) .
$$

We claim that $(\theta, \alpha)$ is a distinguished pair. Observe that if $\gamma$ belonging to $\widetilde{K}$ is such that $\operatorname{deg} \gamma<\operatorname{deg} \alpha$, then $v(\theta-\gamma)<\delta$, for otherwise by the triangle law we would have $v(\alpha-\gamma)>0$ and hence $\bar{\alpha}=\bar{\gamma}$ which is impossible because

$$
m=[\bar{K}(\bar{\alpha}): \bar{K}]=[\bar{K}(\bar{\gamma}): \bar{K}] \leqslant[K(\gamma): K]<m .
$$

So to prove the claim, it suffices to show that whenever $\beta$ belongs to $\widetilde{K}$ with $v(\theta-\beta)>\delta$, then $\operatorname{deg} \beta \geqslant \operatorname{deg} \theta$. For proving this inequality, in view of the fundamental inequality and the fact $\operatorname{deg} \theta=[G(K(\theta)): G][\overline{K(\theta)}: \bar{K}]$ derived from Lemma 3.1, it is enough to show that

$$
G(K(\theta)) \subseteq G(K(\beta)), \overline{K(\theta)} \subseteq \overline{K(\beta)}
$$

Let $\beta$ be an element of $\widetilde{K}$ with $v(\beta-\theta)>\delta$ and $\alpha^{(1)}, \alpha^{(2)}, \ldots, \alpha^{(m)}$ be the roots of $f(x)$, counted with multiplicities, if any. Write

$$
\frac{f(\beta)}{f(\theta)}=\prod_{i=1}^{m}\left(\frac{\beta-\alpha^{(i)}}{\theta-\alpha^{(i)}}\right)=\prod_{i=1}^{m}\left(1+\frac{\beta-\theta}{\theta-\alpha^{(i)}}\right) .
$$

Since $v(\theta-\beta)>\delta$ and by $(7) v\left(\theta-\alpha^{(i)}\right) \leqslant \delta$ for every $i$, it follows from the above expression for $f(\beta) / f(\theta)$ that its $\tilde{v}$-residue equals $\overline{1}$ and hence $v(f(\beta))=v(f(\theta))$. Therefore in view of Lemma 3.1, $G(K(\theta))=G+\mathbb{Z} v(f(\theta)) \subseteq G(K(\beta))$. Also keeping in mind that $v(\theta-\beta)>\delta>0$, we have by Lemma 3.1, $\overline{K(\theta)}=\bar{K}(\bar{\theta})=$ $\bar{K}(\bar{\beta})$ which proves $(8)$ and hence the claim.

Recall that $\bar{\alpha}$ is a root of the irreducible polynomial $\bar{f}(x)$ of degree $m>1$. So $v(\alpha-1)=0$. Also for any $\beta$ in $\widetilde{K}$ with $\operatorname{deg} \beta<\operatorname{deg} \alpha$, we have $v(\alpha-\beta) \leqslant 0$, 
for otherwise $\bar{\alpha}=\bar{\beta}$ and this in view of the fundamental inequality would imply $[K(\beta): K] \geqslant[\bar{K}(\bar{\beta}): \bar{K}]=m$. So $(\alpha, 1)$ is a distinguished pair. Thus we have proved that $\theta$ has a saturated distinguished chain $\theta, \alpha, 1$ of length 2 . Since $[K(\alpha): K]=[\bar{K}(\bar{\alpha}): \bar{K}]=m$, it follows from the fundamental inequality that $G(K(\alpha))=G$. The other two equalities hold by virtue of Lemma 3.1.

\section{PROOF OF THEOREM 1.4.}

We retain the notations of the previous sections as well as the assumption that $v$ is a henselian valuation of arbitrary rank of a field $K$ with unique prolongation $\tilde{v}$ to the algebraic closure $\widetilde{K}$ having value group $\widetilde{G}$. Recall that a pair $(\alpha, \delta)$ belonging to $\widetilde{K} \times \widetilde{G}$ is said to be a minimal pair (more precisely $(K, v)$-minimal pair) if whenever $\beta$ belonging to $\widetilde{K}$ satisfies $\tilde{v}(\alpha-\beta) \geqslant \delta$, then $\operatorname{deg} \beta \geqslant \operatorname{deg} \alpha$. It can be easily seen that if $(\theta, \alpha)$ is a distinguished pair and $\delta=\tilde{v}(\theta-\alpha)$, then $(\alpha, \delta)$ is a minimal pair.

Let $(\alpha, \delta)$ be a $(K, v)$-minimal pair. The valuation $\tilde{w}_{\alpha, \delta}$ of $\widetilde{K}(x)$ defined on $\widetilde{K}[x]$ by

$$
\tilde{w}_{\alpha, \delta}\left(\sum_{i} c_{i}(x-\alpha)^{i}\right)=\min _{i}\left\{\tilde{v}\left(c_{i}\right)+i \delta\right\}, c_{i} \in \widetilde{K}
$$

will be referred to as the valuation defined by the pair $(\alpha, \delta)$. The description of $\tilde{w}_{\alpha, \delta}$ on $K[x]$ is given by the already known theorem stated below (cf. Alexandru, Popescu and Zaharescu, 1988; Khanduja, 1992).

Theorem C. Let $\tilde{w}_{\alpha, \delta}$ be the valuation of $\widetilde{K}(x)$ defined by a minimal pair $(\alpha, \delta)$ and $w_{\alpha, \delta}$ be the valuation of $K(x)$ obtained by restricting $\tilde{w}_{\alpha, \delta}$. Let $f(x)$ be the minimal polynomial of $\alpha$ over $K$. Then for any polynomial $g(x)$ in $K[x]$ with $f(x)$-expansion $\sum_{i \geqslant 0} g_{i}(x) f(x)^{i}$, one has $w_{\alpha, \delta}(g(x))=\min _{i}\left\{\tilde{v}\left(g_{i}(\alpha)\right)+i w_{\alpha, \delta}(f(x))\right\}$.

The following result proved in (cf. Aghigh and Khanduja, 2005, Theorem 1.1(i)) will be used in the sequel.

Lemma D. Let $(\theta, \alpha)$ be a $(K, v)$-distinguished pair and $f(x)$ be the minimal polynomial of $\alpha$ over $K$. Then $G(K(\theta))=G(K(\alpha))+\mathbb{Z} v(f(\theta))$.

Proof of Theorem 1.4. We divide the proof into three steps.

Step $I$. Let $f(x)$ be the minimal polynomial of $\theta_{1}$ over $K$. In this step, we prove that $\bar{f}(x)$ is irreducible over $\bar{K}$ and $\bar{f}(x)=\phi(x)$. In view of the hypothesis $[\overline{K(\theta)}: \bar{K}]=m,[G(K(\theta)): G]=e$ and the fundamental inequality, it follows that $[K(\theta): K] \geqslant \mathrm{em}$. Since $\theta$ is a root of the polynomial $g(x)$ having degree $\mathrm{em}$, 
we have $[K(\theta): K]=e m$. Note that $v\left(\theta-\theta_{1}\right)>0$, because if $F(x) \in R_{v}[x]$ is a monic polynomial with $\bar{F}(x)=\phi(x)$, then there exists a root $\beta$ of $F(x)$ such that $\bar{\beta}=\bar{\theta}$ which in view of the hypothesis $e>1$ implies that $v\left(\theta-\theta_{1}\right) \geqslant v(\theta-\beta)>0$. So the assertion of Step I is proved once we show that

$$
\left[K\left(\theta_{1}\right): K\right]=\left[\bar{K}\left(\overline{\theta_{1}}\right): \bar{K}\right]=m .
$$

Recall that $\overline{K\left(\theta_{1}\right)} \subseteq \overline{K(\theta)}$ by Theorem B. Therefore using the hypothesis $\overline{K(\theta)}=$ $\bar{K}(\bar{\theta})$ and the fact $\overline{\theta_{1}}=\bar{\theta}$, it follows that $\overline{K\left(\theta_{1}\right)}=\bar{K}(\bar{\theta})$; in particular

$$
\left[\overline{K\left(\theta_{1}\right)}: \bar{K}\right]=\left[\bar{K}\left(\overline{\theta_{1}}\right): \bar{K}\right]=m \text {. }
$$

Since $K\left(\theta_{1}\right) / K$ is a defectless extension in view of (Aghigh and Khanduja, 2005, Theorem 1.2) and it is given that $G\left(K\left(\theta_{1}\right)\right)=G$, we now obtain (9) using (10). Step II. For simplicity of notation, we shall henceforth denote $\theta_{1}$ by $\alpha$. Set $v(\theta-\alpha)=\delta$ and $v(f(\theta))=\lambda$. Let $g(x)=f(x)^{e}+\sum_{i=0}^{e-1} g_{i}(x) f(x)^{i}$ be the $f(x)$ expansion of $g(x)$. Let $\tilde{w}_{\alpha, \delta}$ be the valuation of $\widetilde{K}(x)$ defined by the minimal pair $(\alpha, \delta)$. In this step, we prove that

$$
\tilde{w}_{\alpha, \delta}(f(x))=\lambda
$$

and

$$
\tilde{w}_{\alpha, \delta}(g(x))=v^{x}\left(g_{0}(x)\right)=e \lambda .
$$

Write $f(x)=\prod_{i=1}^{m}\left(x-\alpha^{(i)}\right), g(x)=\prod_{j=1}^{e m}\left(x-\theta^{(j)}\right)$. Using the fact that $v\left(\theta-\alpha^{(i)}\right) \leqslant \delta$ and hence $v\left(\theta-\alpha^{(i)}\right)=\min \left\{\delta, v\left(\alpha-\alpha^{(i)}\right)\right\}$, we have

$$
\tilde{w}_{\alpha, \delta}(f(x))=\tilde{w}_{\alpha, \delta}\left(\prod_{i=1}^{m}\left(x-\alpha^{(i)}\right)\right)=\sum_{i=1}^{m} \min \left\{\delta, v\left(\alpha-\alpha^{(i)}\right)\right\}=\sum_{i=1}^{m} v\left(\theta-\alpha^{(i)}\right)=\lambda
$$

which proves $(11)$. Since $(K, v)$ is henselian, for any $K$-conjugate $\theta^{(j)}$ of $\theta$, there exists a $K$-conjugate $\alpha^{(i)}$ of $\alpha$ such that $v\left(\theta^{(j)}-\alpha\right)=v\left(\theta-\alpha^{(i)}\right) \leqslant \delta$; consequently

$$
\tilde{w}_{\alpha, \delta}\left(x-\theta^{(j)}\right)=\min \left\{\delta, v\left(\alpha-\theta^{(j)}\right)\right\}=v\left(\alpha-\theta^{(j)}\right),
$$

which on summing over $j$ gives

$$
\tilde{w}_{\alpha, \delta}(g(x))=v(g(\alpha)) .
$$


Recall that in view of Step I, $\bar{f}(x)$ is irreducible over $\bar{K}$ of degree $m$ having $\bar{\theta}$ as a root. So for any polynomial $A(x)=\sum a_{i} x^{i}$ belonging to $K[x]$ of degree less than $m$, we have

$$
v(A(\theta))=v^{x}(A(x))=\min _{i}\left\{v\left(a_{i}\right)\right\},
$$

for if the above equality does not hold, then $m>1, v(\theta)=0$ and hence the triangle law would imply $v(A(\theta))>\min _{i}\left\{v\left(a_{i} \theta^{i}\right)\right\}=v\left(a_{j}\right)$ (say) and thus $\sum_{i=0}^{m-1} \overline{\left(a_{i} / a_{j}\right)} \bar{\theta}^{i}=\overline{0}$, which is impossible. Keeping in mind the $f(x)$-expansion of $g(x)$ and that $f(\alpha)=0$, we see that $g(\alpha)=g_{0}(\alpha)$ and consequently it follows from (14) that $v(g(\alpha))=v\left(g_{0}(\alpha)\right)=v^{x}\left(g_{0}(x)\right)$ which together with (13) proves the first equality of (12). As $f(x), g(x)$ are irreducible over the henselian valued field $(K, v)$, we have

$$
v\left(f\left(\theta^{(j)}\right)\right)=v(f(\theta)), 1 \leqslant j \leqslant e m, v\left(g\left(\alpha^{(i)}\right)\right)=v(g(\alpha)), 1 \leqslant i \leqslant m .
$$

Keeping in mind that $\prod_{i=1}^{m} g\left(\alpha^{(i)}\right)= \pm \prod_{j=1}^{e m} f\left(\theta^{(j)}\right)$, it is clear from (15) that $v(g(\alpha))=e v(f(\theta))=e \lambda$, which proves the second equality of (12) in view of (13).

Step III. In this step, we prove that $g(x)$ is a Generalized Schönemann polynomial with respect to $v$ and $f(x)$. By Theorem $\mathrm{C},(11)$ and (12), we have

$$
\tilde{w}_{\alpha, \delta}(g(x))=\min _{0 \leqslant i \leqslant e}\left\{v\left(g_{i}(\alpha)\right)+i \lambda\right\}=v^{x}\left(g_{0}(x)\right)=e \lambda .
$$

As $v\left(g_{i}(\alpha)\right)=v^{x}\left(g_{i}(x)\right),(16)$ shows that $v^{x}\left(g_{i}(x)\right)+i \lambda \geqslant e \lambda$ for $0 \leqslant i \leqslant e-1$, i.e.,

$$
\frac{v^{x}\left(g_{i}(x)\right)}{e-i} \geqslant \lambda=\frac{v^{x}\left(g_{0}(x)\right)}{e}>0 .
$$

Recall that $\lambda=v(f(\theta))$. Since $(\theta, \alpha)$ is a distinguished pair, in view of Lemma $\mathrm{D}$ and the hypothesis $G(K(\alpha))=G$, we have

$$
G(K(\theta))=G+\mathbb{Z} \lambda
$$

By hypothesis $[G(K(\theta)): G]=e$, so it follows from (17) that $e$ is the smallest positive integer for which $e \lambda \in G$. This completes the proof of the theorem.

Example 4.1. Let $K$ be the field of 3 -adic numbers with the usual valuation $v_{3}$ whose extension to the algebraic closure $\widetilde{K}$ of $K$ will be denoted by $\tilde{v}_{3}$. 
Consider the polynomial $g(x)=x^{4}+14 x^{2}+1$ with $\bar{g}(x)=\left(x^{2}+1\right)^{2}$. It can be easily seen that $\theta=i(2+\sqrt{3})$ is a root of $g(x)$ where $i=\sqrt{-1}$. Since $\bar{\theta}=\overline{2 i} \notin \bar{K}$ and $\tilde{v}_{3}\left(\theta^{2}-2\right)=1 / 2$, it follows in view of the fundamental inequality that $[K(\theta): K]=4$. A simple calculation shows that the Krasner's constant $\omega_{K}(\theta)=\tilde{v}_{3}(\theta-2 i)=1 / 2$. So by Krasner's Lemma, $\tilde{v}_{3}(\theta-\beta) \leqslant \frac{1}{2}$ for every $\beta \in \widetilde{K}$ with $\operatorname{deg} \beta<4$. Further if for some $\gamma$ in $\widetilde{K}, \tilde{v}_{3}(\theta-\gamma)=\frac{1}{2}$, then $\bar{\theta}=\bar{\gamma}=\overline{2 i}$. Since $\overline{2 i} \notin \bar{K}$, we see that $[K(\gamma): K] \geqslant 2$. Therefore $(\theta, 2 i)$ is a distinguished pair. It can be easily seen that $(2 i, 0)$ is a distinguished pair and hence $\theta, 2 i, 0$ is a saturated distinguished chain for $\theta$ satisfying the hypothesis of Theorem 1.4. So $g(x)$ is a Generalized Schönemann polynomial with respect to $v_{3}$ and $f(x)=x^{2}+4$.

\section{ACKNOWLEDGEMENTS}

The financial support by CSIR (grant no. 09/135(0539)/2008-EMR-I) and National Board for Higher Mathematics, Mumbai is gratefully acknowledged.

\section{REFERENCES}

Aghigh, K., Khanduja, S. K. (2005). On chains associated with elements algebraic over a henselian valued field. Algebra Colloq. 12(4):607-616.

Alexandru, V., Popescu, N., Zaharescu, A. (1988). A theorem of characterization of residual transcendental extensions of a valuation. J. Math. Kyoto Univ 28(4):579-592.

Brown, R. (2008). Roots of generalized Schönemann polynomials in henselian extension fields. Indian J. Pure Appl. Math. 39(5):403-410.

Engler, A. J., Prestel, A. (2005). Valued Fields. Berlin: Springer-Verlag.

Juráš, M. (2006). Eisenstein-Dumas criterion for irreducibility of polynomials and projective transformations of the independent variable. JP J. Algebra, Number Theory and Applications 6(2):221-236.

Khanduja, S. K. (1992). On valuations of $K(x)$. Proc. Edinb. Math. Soc. 35:419-426.

Khanduja, S. K., Saha, J. (1997). On a generalization of Eisenstein's irreducibility criterion. Mathematika 44:37-41.

Popescu, N., Zaharescu, A. (1995). On the structure of the irreducible polynomials over local fields. J. Number Theory 52:98-118.

Ribenboim, P. (1999). The Theory of Classical Valuations. New York: SpringerVerlag. 